\title{
Quando a cultura é considerada nas pesquisas em psicologia do desenvolvimento*
}

\section{When culture enters in developmental psychology}

\author{
Tania Zittoun** \\ Nathalie Muller Mirza*** \\ Anne-Nelly Perret-Clermont****
}

\begin{abstract}
RESUMO
O estudo da "cultura" remete às relações entre "significações coletivas" e "sentido pessoal" do conhecimento e da atividade. Identificamos quatro correntes recentes de pesquisa que têm abordado essas questões, examinamos suas escolhas teóricas e metodológicas, e realçamos pontos importantes para pesquisas futuras.

Palavras-chave: cultura; psicologia do desenvolvimento; significação.
\end{abstract}

\footnotetext{
ABSTRACT

The issue of "culture" addresses the relationship between "collective meaning" and "personal sense" of knowledge and activity. We identify four recent research trends which explored these issues, show their theoretical and methodological choices, and highlight important points for future research.

Key-words: culture; developmental psychology; signification.

* Traduzido por Marta Teixeira do original em francês Quand la culture entre dans les recherches en psychologie du développement, publicado na revista Enfance, n. 2, p. 127-134, 2006.

** Universidade de Lausanne, Suíça.

*** Universidade de Neuchâtel, Suíça.

**** Universidade de Neuchâtel, Suíça.
} Revisão de Tania Stoltz. 
Na psicologia do desenvolvimento proposta por Jérôme Bruner, o problema da "cultura" se traduz pela particular atenção dada aos processos pelos quais uma pessoa torna significativa a situação na qual está envolvida. De fato, na psicologia do desenvolvimento dos últimos cinqüenta anos, o lugar da "cultura" varia paralelamente ao das questões do sentido (privado) e da significação (partilhada) dos conhecimentos, das ações e dos objetos de cultura. ${ }^{1}$ Neste artigo, identificamos quatro tradições de trabalhos que abordam a "cultura" no desenvolvimento. Isso evidencia certos problemas teóricos e metodológicos, e nos permite assinalar certas proposições da psicologia social e cultural do desenvolvimento.

\section{Cultura e significação no desenvolvimento cognitivo}

Foi freqüentemente apontado que a psicologia de Piaget, que procurava estabelecer a gênese das capacidades de pensar no sujeito epistêmico, tinha lidado rápido demais com os fatores sociais e culturais do desenvolvimento. Essa abordagem estimulou pesquisas que Piaget mesmo chamava de suas (Piaget, 1966, p. 12), examinando o desenvolvimento psicológico nas diversas situações sociais e culturais. Os problemas de sentido e significação apareceram aí de duas maneiras paralelas.

\section{A psicologia do desenvolvimento intercultural}

Uma primeira maneira de questionar a universalidade do modelo produzido pelas pesquisas piagetianas consistiu em replicar os testes e as observações em populações muito diferentes das crianças suíças, sujeitos dos trabalhos iniciais. Desde os anos 1960, pesquisadores testam a generalidade das descrições piagetianas em diferentes culturas (ver, por exemplo, BovET, 1968; DASEN, 1974). Todavia, rapidamente, certos problemas aparecem. Bruner, Olver e Greenfield (1966) evidenciam que certos funcionamentos psicológicos privilegiam dimensões icônicas mais que lógicas ou verbais. Outros autores insistem sobre os problemas de tradução, ou de significação ambígua dos objetos utilizados nos testes (Donaldson, 1978; HundeIDE, 1988).

${ }^{1}$ Não pretendemos aqui ser exaustivos nem refazer a história da psicologia cultural - o leitor interessado deverá se reportar principalmente aos textos de Bruner, 1983; Cole, 1996; Shweder, 1990; Valsiner, 1998; Valsiner; Van der Veer, 2000, etc. 
Certos resultados comparativos, sugerindo a ausência de competências ou de desenvolvimento, sublinham fortemente a questão da maneira como os sujeitos compreendem a situação do teste (Auge; Lehalle, 1986; Cole; SCRIBNER, 1974). A descentração tratada pela pesquisa intercultural revela, assim, uma dimensão até aí invisível: a significação da tarefa não é dada em si. A pessoa a quem a tarefa é submetida a interpreta e a (re)constrói, fazendo apelo a sua "cultura pessoal", isto é, às linguagens, às regras e aos modos de pensamento nos quais ela cresceu e aos quais ela tem acesso. É por essa estrutura simbólica que o mundo se revela a ela (VALSINER, 2000), e nesse reservatório simbólico que ela pode buscar recursos para dar sentido às situações inéditas (MulLER MiRZA, 2005a; ZitToun, 2005a, 2005b).

\section{A psicologia social do desenvolvimento}

Outros pesquisadores escolheram interrogar o alcance dos modelos piagetianos sob nossas perspectivas, questionando diretamente a aparente transparência das situações de teste. Entre estas, a tarefa dita de conservação dos líquidos tornou-se paradigmática. ${ }^{2}$

A resposta da criança é classicamente interpretada como reveladora de seu "nível de desenvolvimento operatório". Variando diferentes aspectos dessa situação, pôde-se verificar claramente que crianças que interagiam brevemente com outras crianças, apresentando capacidades iguais ou inferiores, podiam ter progressos espantosos (Doise; Mugny; Perret-Clermont, 1975, 1976; Perret-Clermont, 1979/96; Perret-Clermont; Nicolet, 2001). Além do mais, o fato de que certas crianças de origem social dita modesta sofriam às vezes para dar a resposta desejada ao adulto, até aí interpretado como reflexão de uma "deficiência sociocultural", revelou ser freqüentemente o resultado de um mal-entendido sobre a tarefa e suas proposições (Mugny; Perret-Clermont, 1985; Perret-Clermont, 1979/96; Perret-Clermont; Schubauer-Leoni, 1981). A resposta que as crianças dão a essas tarefas parece estar ligada à maneira com a qual elas dão sentido à situação, suas regras, o papel que lhes é atribuído, e os anseios dos adultos (Grossen, 1988; Light, 1986). Isso evidencia novamente a estreita relação entre a avaliação das capacidades de raciocínio e a maneira como os participantes se entendem sobre a significação e as proposições da situação de avaliação.

2 Nesta tarefa, o experimentador transvasa o mesmo conteúdo de líquido de um copo comprido e estreito para um copo largo e baixo; a criança deve então responder "se há sempre o mesmo tanto de líquido nos dois copos, ou se há menos, ou se há mais". A criança "conservadora" tem a capacidade de ultrapassar a ilusão perceptiva da desigualdade, e relacionar a compensação do aumento ou diminuição da altura do nível do conteúdo com sua largura. 


\section{As psicologias sociais e culturais do desenvolvimento}

O problema da significação das situações tornou-se igualmente central nas tradições que se desenvolveram após a difusão na América do Norte e na Europa, a saber, os trabalhos de Luria (1979), Vygotsky (1930-1934/1962, 1987, 1994), Leont'ev (1978) e Bakhtin (1984). Esses autores apreendem o desenvolvimento do pensamento quando mediado por "instrumentos culturais" (objetos materiais e signos), constituídos historicamente por um dado grupo. $\mathrm{O}$ uso desses instrumentos permite às sociedades se desenvolver, e aos indivíduos controlar o seu meio, assim como regular e desenvolver seus pensamentos. Esses autores examinam o papel desses instrumentos no raciocínio (Luria), e alcançam a articulação da consciência individual e da atividade coletiva (Leont'ev). É Leont'ev (1978) quem desenvolve uma distinção já evocada por Vygotsky (1934) entre, de um lado, a significação social, partilhada das ações dos objetos, e, do outro, o sentido mais pessoal e privado que estes podem adquirir, e que se ancora na história e na subjetividade de cada um. Enfocamos quatro direções de trabalhos que deram continuidade a esses trabalhos.

\section{A narração e as obras culturais}

Um dos primeiros editores de Vygotsky em inglês (1962), Jérôme S. Bruner, logo se propôs a completar o estudo do desenvolvimento cognitivo por um exame dos processos de construção de sentido e de significações (BRUNER, 1960/1996, p. 17-18). Seus trabalhos referiram-se à aprendizagem e à educação, e ao sentido atribuído pelos alunos aos saberes em jogo (BRUNER, 1960, 1983a, 1996). Nesses estudos sobre a transmissão e a aquisição da linguagem, Bruner evidencia os formatos da conversação que permitem à criança se apropriar de modelos de relações e de significações (BRUNER, 1983b, 1987; FELDMAN et al., 1994). Ele mostra como a criança e o adulto dão sentido às suas vivências e constroem assim uma identidade graças a competências narrativas, permitindo contar histórias a partir de uma série mais ou menos caótica de incidentes.

Metodologicamente, é o discurso das interações adultos-crianças, depois autobiográfico (BRUNER, 1990), literário (BRUNER, 2002) ou legal (AMSTERDAM; BRUNER, 2000), que é objeto de análise.

Nesses trabalhos, o problema do desenvolvimento torna-se, então, ligado àquele da construção da significação e do sentido: os objetos de conhecimento tomam sentidos específicos para as pessoas e os grupos, em função dos percursos e dos seus saberes, em situações específicas. 


\section{A atividade}

Nos trabalhos de Michael Cole (1996), que igualmente teve um papel muito importante na difusão dos psicólogos russos no mundo anglo-saxão, a cultura é principalmente examinada em termos de artefatos mediando as atividades das pessoas. Scribner e Cole (1981) desenvolveram a noção de atividade como lugar onde se constituem e se mobilizam os conhecimentos; Cole examinou principalmente o papel das mediações informáticas na emergência de novas atividades. Paralelamente, James Wertsch $(1991,1998)$ propõe uma linha de trabalhos originais sobre o papel dos instrumentos culturais, atualmente orientados para questões de memória coletiva (WERTSCH, 2002). Outros trabalhos (LAVE; Chaiklin, 1993; Rogoff, 2003; SCRIBNER, 1984) evidenciam a importância do contexto (concreto, social e histórico) e das práticas cotidianas concretas para compreender os processos cognitivos atualizados.

Nessa linha de pesquisa, não são tanto as significações "na cabeça" das pessoas, mas as significações negociadas ao longo do desdobramento de práticas que são observadas (Muller Mirza, 2005a).

A questão do desenvolvimento é aqui secundária e aparece essencialmente sob a perspectiva da socialização e da participação competente nas atividades.

\section{Os processos semióticos}

Uma outra linha de trabalhos se desenvolveu seguindo a leitura de Vygotsky proposta por Jean Valsiner (1998). Aqui, o problema não é tanto examinar as atividades ou a linguagem, mas interrogar-se sobre a natureza basicamente semiótica das atividades, pensamentos e comunicações humanas.

Metodologicamente, situações quase experimentais ou entrevistas são criadas. A linguagem é considerada como processo de externalização de uma pessoa, sobre a base das formas culturais que ela internalizou, sua "cultura pessoal" (VALSINER, 2000). Assim, o trabalho do sentido se revela indiretamente "entre" significações partilhadas ou canônicas de certos objetos culturais, e o que as pessoas dizem ou fazem delas (ABBey, 2006; LAWrence; Valsiner, 2003; ZitToun, 2005b).

Esses trabalhos repousam sobre a idéia da irreversibilidade do tempo, e têm por objeto a emergência de novas formas de conduta; nesse sentido, eles são eminentemente desenvolvimentistas. 


\section{Perspectivas dialógicas}

Uma quarta linha de trabalhos se desenvolveu no cruzamento dessas tradições e dos trabalhos pós-piagetianos. Certos autores enriqueceram uma visão do desenvolvimento cognitivo da criança voltando sua atenção para as proposições sociais e culturais. Eles examinam as interações inscritas em estruturas sociais preexistentes, que fazem com que as pessoas negociem e construam não somente conhecimentos, mas também suas posições mútuas (Grossen; Liengme Bessire; Perret-Clermont, 1997; Pontecorvo, 2004).

Outros trabalhos estudam a mudança das pessoas nas atividades situadas - em aula, no trabalho - prestando atenção nos discursos e nas práticas que se desdobram ao longo das atividades (CLÔT, 1999; RochEX, 1999), para evidenciar as significações partilhadas, que emergem, são negociadas, se cristalizam ou se transformam (Muller; Perret-Clermont, 1999).

Metodologicamente, os autores se propõem a examinar esses processos em tempo real, nos lugares onde eles se desenrolam naturalmente. A linguagem torna-se para alguns um meio de fazer inferências sobre as modificações do raciocínio das pessoas (MARRO et al., 1999; TROGNON, 1994); para outros, ela torna-se acompanhamento das formas de comunicação não-verbais, e é, ao mesmo tempo, produto e meio de mudança das estruturas sociais e das pessoas (Moro; Rickenmann, 2004).

Assim, aqui também, a questão do desenvolvimento se estende bem além da infância na questão da constante evolução das relações interpessoais, das práticas e de significações partilhadas.

\section{Quais psicologias do desenvolvimento?}

Este breve exame da consideração da cultura na pesquisa em psicologia do desenvolvimento mostra a onipresença das questões de sentido e de significação. Essas questões levaram a um deslocamento da questão do desenvolvimento. Não são mais as crianças, adquirindo uma sucessão de competências práticas e cognitivas tornando-as adultos, que são estudadas. A pesquisa em desenvolvimento examina, no momento, pessoas de todas as idades, fazendo constantemente a experiência de situações inéditas, e para as quais elas produzem (às vezes) novas condutas. A mudança, o desenvolvimento, surge, então, no espaço de tensões, de fricções entre as significações partilhadas das coisas e o sentido único e sempre por reinventar que lhes atribuem as pessoas.

Conseqüentemente, os dispositivos metodológicos foram igualmente modificados. Os pesquisadores reconhecem que o trabalho de construção 
de sentido ou de negociação de significações depende da complexidade de numerosas dimensões; ele pode ser alcançado por diversos caminhos, e sua evolução é, em parte, imprevisível. Assim, os pesquisadores, com freqüência, renunciaram ao controle estrito de certas variáveis, e procuraram estabelecer métodos de coleta de dados compatíveis com sua compreensão do que pode ser a cultura, tomando principalmente em consideração as atividades cotidianas nas quais as pessoas estão envolvidas.

Todavia, a flexibilidade da noção de desenvolvimento e as transformações metodológicas ligadas à atenção crescente com respeito à "cultura" não devem nos fazer esquecer as questões de poder, de valor e de identidade que atravessam e transformam as situações de aprendizagem, ensino ou avaliação. Principalmente os trabalhos da psicologia dita "social do desenvolvimento" mostraram as colocações interpessoais de reconhecimento e de posicionamento, os efeitos de enquadramento e regras, de estruturas e forças sociais, de representações sociais e de crenças, na situação da aprendizagem. Esses trabalhos igualmente desenvolveram uma análise específica do papel do pesquisador nas situações de coleta e análise de dados, o que sinaliza um alerta à construção das respostas e dos resultados da pesquisa na interação entre pesquisador e sujeito (Doise; Mugny, 1997; Duveen; Lloyd, 1990; Grossen, 1988; MonteIL, 1997; Muller Mirza, 2005b; Nicolet; Grossen; Perret-Clermont, 1988; Psaltis; Duveen, 2005). Para evitar uma visão por demais "romântica" do papel da cultura no desenvolvimento, nos parece importante preservar uma atenção crítica às dimensões sociais e societárias da situação de pesquisa.

Desse breve olhar de diferentes trabalhos quase sempre descoordenados, podemos reter alguns fundamentos para a pesquisa futura. Para evitar novamente a perda da "cultura" na pesquisa em desenvolvimento, trata-se de levar em conta sua natureza constitutiva, estruturante de situações locais e de interações interpessoais, e considerá-la em seus aspectos semióticos e intrapsíquicos. Trata-se, então, de não perder de vista a tensão criativa entre sentido pessoal e significações partilhadas dos objetos de cultura.

\section{REFERÊNCIAS}

ABBEY, E. Perceptual uncertainty of cultural life: becoming reality. In: VALSINER, J.; ROSA, A. R. (Orgs.). Handbook of sociocultural psychology. Cambridge: Cambridge University Press, 2006. 
AMSTERDAM, A.; BRUNER, J. S. Minding the law. Cambridge, MA: Harvard University Press, 2000.

AUGE, C. ; LEHALLE, H. Effets de 'signification' et conservation du volume. Enfance, n. 1, p. $43-51,1986$.

BAKHTIN, M. Esthétique de la création verbale. Paris: Gallimard, 1984.

BOVET, M. Études interculturelles du développement intellectuel. Revue Suisse de Psychologie, n. 27, p. 189-199, 1968.

BRIL, B.; LEHALLE, H. Le développement psychologique est-il universel? Approches interculturelles. Paris: Presses Universitaires de France, 1988.

BRUNER, J. S. The process of education. Cambridge, Mass.; London, UK: Harvard University Press, 1960/1999.

1983a.

. In search of mind: essays in autobiography. New York, NY: Harper and Row, . Le développement de l'enfant: savoir faire, savoir dire. Paris: PUF, $1983 \mathrm{~b}$.

. Comment les enfants apprennent à parler. Paris: Retz, 1987.

. Acts of meaning. Cambridge: Harvard University Press, 1990.

. L'éducation, entrée dans la culture. Paris: Retz, 1996.

. Pourquoi nous racontons-nous des histoires? Paris: Retz, 2002.

BRUNER, J. S. et al. Studies in cognitive growth. New-York: J. Wiley, 1966.

CLOT, Y. (Org.). Avec Vygotsky. Paris: La Dispute, 1999.

COLE, M.; SCRIBNER, S. Culture and thought, a psychological introduction. New York: John Wiley, 1974.

COLE, M. Cultural psychology. A once and future discipline. Cambridge: The Belknap Press of Harvard University Press, 1996.

DASEN, P. R. Cross-cultural piagetian research: a summary. In: BERRY, J. W.; DASEN, P. R. (Orgs.). Culture and cognition: readings in cross-cultural psychology. London: Methuen and Co, 1974.

DOISE, W.; MUGNY, G. Psychologie sociale et développement cognitif. Paris: Armand Colin, 1997. 
ZITTOUN, T.; MIRZA, N. M.; PERRET-CLERMONT, A. Quando a cultura é considerada nas pesquisas em psicologia do desenvolvimento

DOISE, W.; MUGNY, G.; PERRET-CLERMONT, A.-N. Social interaction and the development of cognitive operation. European Journal of Social Psychology, v. 5, n. 3, p. 367-383, 1975.

. Social interaction and the development: further evidence. European Journal of Social Psychology, v. 6, n. 2, p. 245-247, 1976.

DONALDSON, M. Children's minds. New York: Norton, 1978.

DUVEEN, G.; LLOYD, B. Social representations and the development of knowledge. Cambridge: Cambridge University Press, 1990.

FELDMAN, C. et al. Plot, plight and dramatism: interpretation at three ages. In: OVERTON, W.-F.; PALERMO, D.-S. (Orgs.). The nature and ontogenesis of meaning. Hillsdale: Lawrence Erlbaum Associates, 1994. p. 255-279.

GROSSEN, M. La construction de l'intersubjectivité en situation de test. Fribourg (Cousset): DelVal; Neuchâtel: Dossiers de Psychologie de l'Université de Neuchâtel, 1988.

GROSSEN, M.; LIENGME BESSIRE, M.-J.; PERRET-CLERMONT, A.-N. Construction de l'interaction et dynamiques socio-cognitives. In: GROSSEN, M.; PY, B. (Orgs.). Pratiques sociales et médiations symboliques. Bern: Peter Lang, 1997. p. 221-247.

HUNDEIDE, K. Metacontracts for situational definitions and for presentation of cognitive skills. The Quarterly Newsletter of the Laboratory of Comparative Human Cognition, v. 10, n. 3, p. 85-91, 1988.

LAVE, J.; CHAIKLIN, S. (Orgs.). Understanding practice: perspectives on activity and context. Cambridge: University Press, 1993.

LAWRENCE, J.; VALSINER, J. Making personal sense. An account of basic internalisation and externalisation processes. Theory \& Psychology, v. 13, n. 6, p. 723-752, 2003.

LEONTEV, A. N. Activity, consciousness, and personality. 1978. Disponível em: $<$ http://www.marxists.org/archive/leontev/works/1978/index.htm>.

LIGHT, P. Context, conservation and conversation. In: RICHARD, P. L. M. (Org.). Children of social worlds. Development in a social context. Cambridge: Polity Press, 1986.

LURIA, A. R. The making of mind. A personal account of Soviet psychology. Cambridge, MA/London: Harvard University Press, 1979. 
MARRO CLEMENT, P.; TROGNON, A.; PERRET-CLERMONT, A.-N. Processus interloctoires dans une tâche de conservation des liquides. In: GILLY, M.; ROUX, J.-P.; TROGNON, A. (Orgs.). Apprendre dans l'interaction. Nancy: Presses Universitaire de Nancy, 1999. p. 163-180.

MONTEIL, J.-M. Éduquer et former. Perspectives psycho-sociales. Grenoble: Presses Universitaires de Grenoble, 1997.

MORO, V.; RICKENMANN, R. (Orgs.). Situation éducative et significations. Bruxelles: De Boeck, 2004.

MUGNY, G.; PERRET-CLERMONT, A.-N. Effets sociologiques et processus didactique. In: MUGNY, G. (Ed.). Psychologie sociale du développement cognitif. Berne: Editions Peter Lang, 1985. p. 251-261.

MULLER MIRZA, N. Psychologie culturelle d'une formation d'adulte. L'île aux savoirs voyageurs. Paris: L'Harmattan, 2005a.

. How to take into account asymetries? A tentative in cultural psychology. ISCAR Conference (Séville), $2005 \mathrm{~b}$.

MULLER, N.; PERRET-CLERMONT, A.-N. Negotiating identities and meanings in the transmission of knowledge: analysis of interactions in the context of a knowledge exchange network. In: BLISS, J.; SÄLJÖ, R.; LIGHT, P. (Orgs.). Learning sites, social and technological resources for learning. Earli: Pergamon, 1999. p. 47-60.

NICOLET, M.; GROSSEN, M.; PERRET-CLERMONT, A.-N. Testons-nous des compétences cognitives. Revue Internacionale de Psychologie Sociale, n. 1, p. 72-91, 1988.

PERRET-CLERMONT, A.-N. La construction de l'intelligence dans l'interaction sociale. Berne: Peter Lang, édition revue et augmentée, 1979/1996.

PERRET-CLERMONT, A.-N.; NICOLET, M. Interagir et connaître. Paris: L'Harmattan, 2001.

PERRET-CLERMONT, A.-N.; SCHUBAUER-LEONI, M.-L. Conflict and cooperation on opportunities for learning. In: ROBINSON, W. P. (Org.). Communication in development. London: Academic Press, 1981. p. 203-233.

PERRET-CLERMONT, A.-N.; PERRET, J.-F.; BELL, N. The social construction of meaning and cognitive activity in elementary school children. In: RESNICK, L. B.; LEVINE, J. M.; TEASLEY, S. D. (Orgs.). Perspectives on socially shared cognition. Washington, D.C.: American Psychological Association, 1991. p. 41-62. 
PIAGET, J. Nécessité et signification des recherches comparatives en psychologie génétique. International Journal of Psychology, n. 1, p. 3-13, 1966.

PONTECORVO, C. Thinking with others: the social dimensions of learning in families and schools. In: PERRET-CLERMONT, A.-N. et al. (Orgs.). Joining society: social interactions and learning in adolescence and youth. New York/Cambridge: Cambridge University Press, 2004. p. 227-240.

PSALTIS, C.; DUVEEN, G. Social relations and cognitive development: the influence of conversation type and representations of gender. European Journal of Social Psychology, 2005, in press.

ROCHEX, J.-Y. Le sens de l'experience scolaire. Paris: Presses Universitaires de France, 1999.

ROGOFF, B. The cultural nature of human develpment. New York: Oxford University Press, 2003.

SCRIBNER, S.; COLE, M. The psychology of literacy. Cambridge, MA: Harvard University Press, 1981.

SCRIBNER, S. Studying working intelligence. Cambridge. MA: Harvard University Press, 1984.

SHWEDER, R. A. Cultural psychology: what is it? In: STIGLER, J.; SHWEDER, R. A.; HERDT, G. (Orgs.). Cultural psychology: essays on comparative human development. New York: Cambridge University Press, 1990. p. 1-46.

TROGNON, A. Théories et modèles de la construction interactive. Nancy: Presses Universitaire de Nancy, 1994.

VALSINER, J. The guided mind. A sociogenetic approach to personality. Cambridge (Mass); London: Harvard University Press, 1998.

. Culture and human development. London/Thousand Oaks: Sage, 2000.

VALSINER, J.; VAN DER VEER, R. The social mind: construction of the idea. Cambridge: Cambridge University Press, 2000.

VYGOTSKY, L. S. Thought and language (com prefácio de J. S. Bruner). Cambridge, MA: The MIT Press, 1934/1962. (Tradução em francês por SÈVE, F. Pensée et langage. Paris: La Dispute, 1997).

. The collected works of Vygotsky. New York: Plenum Press, 1987. 
ZITTOUN, T.; MIRZA, N. M.; PERRET-CLERMONT, A. Quando a cultura é considerada nas pesquisas em psicologia do desenvolvimento

WERTSCH, J. V. Voices of the mind. A sociocultural approach to mediated action. Cambridge, MA: Harvard University Press, 1991. . Mind as action. New York: Oxford University Press, 1998. 2002. .Voices of collective remembering. New York: Cambridge University Press,

ZITTOUN, T. Donner la vie, choisir un nom. Engendrements symboliques. Paris: L'Harmattan, 2005a.

. Transitions. Development through symbolic resources. Greenwich (CT): InfoAge, 2005b.

Texto recebido em 23 abr. 2006

Texto aprovado em 19 ago. 2006 